\title{
Propriedades da Madeira de Eucalyptus umbra R. T. Baker em Função do Diâmetro e da Posição Radial na Tora
}

\author{
Israel Luiz de Lima ${ }^{1}$, Eduardo Luiz Longui ${ }^{1}$, Rafael Garcia ${ }^{1}$, \\ Edgar Fernando de Luca ${ }^{1}$, Francides Gomes da Silva Júnior ${ }^{2}$, \\ Sandra Monteiro Borges Florsheim ${ }^{1}$ \\ ${ }^{1}$ Instituto Florestal do Estado de São Paulo - IF \\ ${ }^{2}$ Departamento de Ciências Florestais, Escola Superior de Agricultura "Luiz de Queiroz" - ESALQ, \\ Universidade de São Paulo - USP
}

\begin{abstract}
RESUMO
Neste trabalho, investigou-se a variação da densidade básica, dos constituintes químicos e das características anatômicas em função da classe de diâmetro (superior, mediana e inferior) e posição radial nas árvores de uma população de Eucalyptus umbra, de 25 anos de idade. Apenas o comprimento das fibras variou entre as classes de diâmetro. Fibras mais longas ocorreram na classe superior e fibras mais curtas, na classe inferior. Os resultados mostram a homogeneidade da madeira entre as classes de diâmetro. A largura e a frequência de raio, bem como o teor de lignina, não foram influenciadas significativamente pela posição radial. O comprimento de fibras e os elementos de vaso, assim como o diâmetro dos vasos, aumentaram significativamente no sentido medula-casca. A partir das análises de regressão, concluiu-se que a variação radial é decorrente da maturação do câmbio vascular e da transição de lenho juvenil para lenho adulto.
\end{abstract}

Palavras-chave: Eucalyptus, densidade básica, características anatômicas, composição química.

\section{Properties of the Eucalyptus umbra R. T. Baker Wood as a Function of the Diameter and Radial Position in the Log}

\begin{abstract}
The purpose of this study was to investigate the variation of density, chemical compounds and anatomical characteristics, as a function of the diameter class (upper, middle and lower) and radial position, in the trees of Eucalytpus umbra in a 25-year-old population. Only the fiber length varied throughout diameter classes: longer fibers occurred in the upper class and shorter fibers in the lower class. Results showed homogeneity of the wood in the diameter classes. Ray width, frequency and lignin content were not affected by the radial position. Fiber and vessel length and vessel diameter increased significantly from pith to bark. Through regression analysis, it was concluded that the radial variation occurs due to vascular cambium maturation and the transition from juvenile to adult wood.
\end{abstract}

Keywords: Eucalyptus, basic density, anatomical characteristics, chemical composition. 


\section{INTRODUÇÃO}

Eucalyptus umbra ocorre naturalmente no norte de Bundaberg e ao Sul de Sidney, na Austrália, ocupando uma faixa de $200 \mathrm{~km}$ da costa (Hall \& Brooker, 1977). A madeira dessa espécie é empregada na produção de lenha, polpa celulósica e, por apresentar boa qualidade, é usada para uso geral; além disso, pode ser indicada para formação de quebra-vento e, por causa da sua beleza, as árvores servem para projetos paisagísticos em regiões de clima subtropical de altitude (Lorenzi et al., 2003).

Segundo Latorraca \& Alburquerque (2000), os fatores que afetam as propriedades da madeira podem ser inerentes à própria madeira e ao ambiente em que a árvore se desenvolve. Para Varghese et al. (2000), as propriedades das madeiras podem ser influenciadas diferentemente pela taxa de crescimento das árvores.

Trevisan et al. (2007) verificaram que a densidade básica das árvores da classe média de uma população de Eucalyptus grandis foi influenciada pelo desbaste, porém sem apresentar tendência clara em relação à intensidade da intervenção; verificou-se, ainda, que a produção de massa seca das árvores médias aumentou com a intensidade do desbaste e que não afetou a produção das árvores dominantes.

A maioria das espécies madeireiras apresenta variações nas características anatômicas, físicas, mecânicas e químicas no tronco das árvores, no sentido medula-casca; na maioria das vezes, essas variações são maiores nesse sentido do que da base para o topo (Cruz et al.,2003; Lima \& Garcia, 2010). Essas variações são decorrentes de mudanças progressivas no câmbio vascular e, consequentemente, nas dimensões e frequências das células por ele geradas, na deposição da parede celular e na formação e no acúmulo de extrativos, o que pode influenciar a maioria das propriedades das madeiras (Panshin \& de Zeeuw, 1964; Zobel \& Buijtenen, 1989; Longui, 2005).

A despeito do grande potencial de uso da madeira de E. umbra, são raras as referências na literatura sobre as suas propriedades e indicação de uso. Nesse contexto, o objetivo do presente trabalho foi estudar as variações de propriedades da madeira de Eucalyptus umbra, em função da posição radial e de diferentes classes de diâmetro.

\section{MATERIAL E MÉTODOS}

As amostras de madeira foram coletadas de um povoamento de Eucalyptus umbra situado na Estação Experimental de Tupi, localizada em Piracicaba-SP (Latitude $21^{\circ} 22^{\prime} \mathrm{S}$ e longitude $48^{\circ} 03^{\prime} \mathrm{W}$ e altitude $600 \mathrm{~m})$. De acordo com a classificação de Köppen, o clima é do tipo Cwa: caracterizado pelo clima tropical de altitude, com chuvas no verão e seca no inverno.

O povoamento de E. umbra foi plantado em 1984, com espaçamento de $3 \times 2 \mathrm{~m}$, totalizando dessa forma 1667 árvores por hectare. A partir de 1996, foram realizados desbastes seletivos visando, principalmente, madeira para ser usada como mourões. Atualmente, a população contava com 716 árvores.ha ${ }^{-1}$, cujos DAP médio e altura média eram $21,9 \mathrm{~cm}$ e $18,6 \mathrm{~m}$, respectivamente.

A Figura 1 mostra a distribuição diamétrica da população de E. umbra.

De acordo com a distribuição diamétrica, aos 25 anos de idade da população, foram eleitas três classes de DAP para este estudo: classe inferior (DAP entre 22 e $26 \mathrm{~cm}$ ), classe mediana (DAP entre $26 \mathrm{e}$ $30 \mathrm{~cm}$ ) e classe superior (DAP entre 30 e $34 \mathrm{~cm}$ ). Dentro de cada uma das classes, foram tomadas três árvores, que foram devidamente identificadas. Em cada uma delas, retirou-se um disco de $7 \mathrm{~cm}$ de espessura na região do DAP e dele tiraram-se amostras representativas das posições $0,25,50,75 \mathrm{e}$ $100 \%$ no sentido medula-casca, para a obtenção dos corpos de prova $(2 \times 2 \times 3 \mathrm{~cm})$. As propriedades estudadas foram: densidade básica; teor de extrativos, lignina e holocelulose; comprimento e espessura

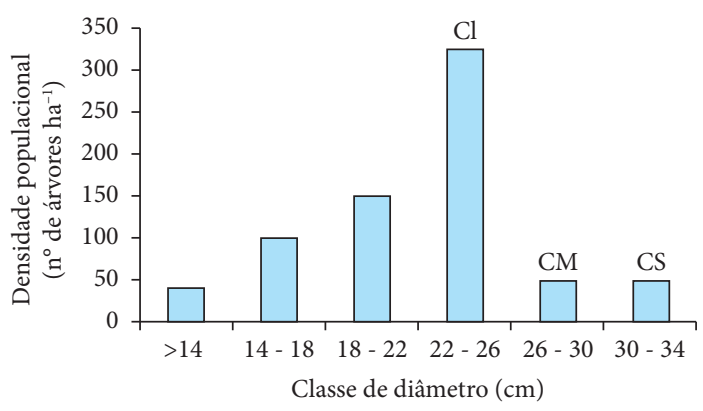

Figura 1. Distribuição diamétrica em Eucalyptus umbra aos 25 anos de idade.

Figure 1. Diametric distribution in Eucalyptus umbra of 25-years-old. 
da parede das fibras; comprimento, diâmetro e frequência dos elementos de vasos; frequência, altura e largura dos raios.

Na obtenção da densidade básica (DB), utilizouse o método da balança hidrostática, conforme Vital (1984). Os corpos de prova foram saturados em água por um período de aproximadamente um mês, possibilitando a obtenção da massa saturada. Posteriormente, os mesmos foram secos em estufa até atingirem a massa seca constante a $105 \pm 3{ }^{\circ} \mathrm{C}$.

Para a análise das características químicas, foram retiradas amostras dos corpos de prova que foram fragmentadas e moídas. O pó resultante foi classificado em peneiras de malha 40 e 60 mesh, sendo selecionado o material retido na última peneira. Foram empregadas as normas TAPPI (The Leading..., 1998), sendo TAPPI T20 e T222 para determinar os teores de extrativos e de lignina, respectivamente; o teor de holocelulose foi obtido por diferença a partir da Equação 1:

$$
H O=[100-(E T+L T)]
$$

em que: $\mathrm{HO}=$ holocelulose (\%); $\mathrm{ET}=$ extrativos (\%); $\mathrm{LT}=$ lignina $(\%)$.

Para as análises anatômicas, de cada amostra obtiveram-se corpos de prova com cerca de $2 \mathrm{~cm}^{3}$ $\mathrm{e}$, destes, foram retirados pequenos fragmentos que foram macerados em solução de ácido acético e peróxido de hidrogênio 1:1, sendo, posteriormente, corados com safranina alcoólica a 1\% (Berlyn \& Miksche, 1976). Os mesmos corpos de prova foram amolecidos em solução de água, álcool e glicerina, em partes iguais, e posteriormente seccionados em micrótomo de deslize Zeiss Hyrax S50, com espessura entre 12 e $25 \mu \mathrm{m}$. Seções de cada amostra foram clarificadas com hipoclorito de sódio a $60 \% \mathrm{e}$ coradas com safranina 1\% (Sass, 1951). Prepararamse lâminas provisórias para a análise e a mensuração das características anatômicas, que foram realizadas de acordo com o IAWA Committee (International..., 1989). Para calcular a espessura da parede das fibras, utilizou-se a Equação 2:

$$
E P F=\frac{D F-L F}{2}
$$

em que: $\mathrm{EPF}=$ espessura da parede da fibra; $\mathrm{DF}=$ diâmetro da fibra; $\mathrm{LF}=$ diâmetro do lume.
A caracterização microscópica e as mensurações das células foram realizadas em microscópio da marca Olympus modelo CX 31, equipado com câmera digital Olympus EVOLT E-330 e computador com software de análise de imagens Image - Pro Plus versão 6.3. Para amostrar todas as dimensões celulares, foi adotado $\mathrm{n}=25$ medições.

As hipóteses testadas foram: a) a classe de diâmetro não influencia a densidade básica, as características químicas e as dimensões celulares da madeira de E. umbra de 25 anos de idade; b) não existem variações para: densidade básica, características químicas e dimensões celulares na madeira na direção da casca em E. umbra de 25 anos de idade.

Para a análise estatística dos dados, inicialmente empregou-se o teste de homogeneidade de variância, utilizando-se o teste de Hartley. Posteriormente, foi feito o teste $\mathrm{F}$ de análise de variância, obedecendo-se ao delineamento experimental inteiramente ao acaso e adotando-se o esquema fatorial $3 \times 5$ (classes $\times$ posição radial) para o estudo das propriedades. Utilizou-se o teste de Tukey para comparação de médias quando o teste $\mathrm{F}$ foi significativo a $5 \%$ de significância. Foi realizado também um estudo da relação entre densidade básica e características químicas e dimensões das fibras, com a posição radial; para tanto, foi utilizada análise de regressão, em nível de probabilidade de $95 \%$. A análise dos dados foi feita utilizando-se os procedimentos PROC GLM e PROC REG do programa estatístico SAS (SAS, 1999).

\section{RESULTADOS E DISCUSSÃO}

Na Tabela 1, apresenta-se o resumo da análise de variância das propriedades em estudo da madeira de E. umbra, aos 25 anos de idade, em função de cada característica. Pode-se observar que a classe de diâmetro influenciou, significativamente, somente o comprimento de fibra, enquanto que a posição radial influenciou, significativamente, quase todas as variáveis em estudo, com exceção da largura e da altura de raio e do teor de lignina.

De acordo com o exposto, a densidade básica não foi influenciada pela classe diamétrica, ou seja, a diferença no crescimento das árvores, em função 
Tabela 1. Resumo da análise de variância efetuada para densidade básica (DB), comprimento das fibras (CF), espessura da parede das fibras (EPF), comprimentos dos elementos de vaso (CV), diâmetro dos vasos (DV), frequência dos vasos (FV), altura dos raios (AR), largura dos raios (LR), frequência dos raios (FR), teor de extrativo (TE), teor de lignina (TL) e teor de holocelulose (TH) de Eucalyptus umbra.

Table 1. Summary of analysis of variance of basic density (DB), fiber length (CF), fiber wall thickness (EPF), vessel element length $(\mathrm{CV})$, vessel diameter (DV), vessel frequency (FV), ray height (AR), ray width (LR), ray frequency (FR), extractive content (TE), lignin content (TL) and holocellulose content (TH) of Eucalyptus umbra.

\begin{tabular}{|c|c|c|c|c|c|c|c|c|c|c|c|c|c|}
\hline \multirow[b]{2}{*}{$\begin{array}{l}\text { Causa de } \\
\text { variação }\end{array}$} & \multirow[b]{2}{*}{ GL } & \multirow[b]{2}{*}{$\underset{\left(\mathbf{k g} \cdot \mathrm{m}^{-3}\right)}{\mathrm{DB}}$} & \multirow[b]{2}{*}{$\begin{array}{c}\mathrm{CF} \\
(\mu \mathrm{m})\end{array}$} & \multirow[b]{2}{*}{$\begin{array}{l}\text { EPF } \\
(\mu \mathrm{m})\end{array}$} & \multirow[b]{2}{*}{$\begin{array}{c}\mathrm{CV} \\
(\mu \mathrm{m})\end{array}$} & \multicolumn{8}{|c|}{ Quadrado médio } \\
\hline & & & & & & $\begin{array}{c}\mathrm{DV} \\
(\mu \mathrm{m})\end{array}$ & $\begin{array}{c}\mathbf{F V} \\
\left(\mathbf{n}^{\mathbf{0}} \cdot \mathbf{m m}^{-2}\right)\end{array}$ & $\begin{array}{c}\text { AR } \\
(\mu \mathrm{m})\end{array}$ & $\begin{array}{c}\text { LR } \\
(\mu \mathrm{m})\end{array}$ & $\begin{array}{c}\text { FR } \\
\left(\mathbf{n}^{\mathbf{0}} \cdot \mathbf{m m}^{-1}\right)\end{array}$ & $\begin{array}{l}\text { TE } \\
(\%)\end{array}$ & $\begin{array}{c}\text { TL } \\
(\%)\end{array}$ & $\begin{array}{l}\text { TH } \\
(\%)\end{array}$ \\
\hline Classe (C) & 2 & $1421^{\mathrm{n} . \mathrm{s}}$ & $41686^{*}$ & $1,1^{\mathrm{n} . \mathrm{s}}$ & $9226^{\text {n.s }}$ & $495^{\text {n.s }}$ & $3,64^{\mathrm{n} . \mathrm{s}}$ & $1198^{\text {n.s }}$ & $31,6^{\text {n.s }}$ & $0,3^{\text {n.s }}$ & $17,2^{\mathrm{n} . \mathrm{s}}$ & $3,2^{\text {n.s }}$ & $5,5^{\mathrm{n.s}}$ \\
\hline $\begin{array}{l}\text { Posição } \\
\text { radial (R) }\end{array}$ & 4 & $72844^{* *}$ & $236197^{\star *}$ & $6,7^{* *}$ & $5450^{* *}$ & $4921^{* *}$ & $138,90^{* *}$ & $263^{\text {n.s. }}$ & $8,3^{\text {n.s. }}$ & $7,5^{* *}$ & $33,3^{* *}$ & $10,6^{\text {n.s. }}$ & $22,8^{* *}$ \\
\hline $\mathrm{C} \times \mathrm{R}$ & 8 & $2770^{\mathrm{n} . \mathrm{s}}$ & $9410^{\text {n.s }}$ & $1,2^{\mathrm{n} . \mathrm{s}}$ & $4843^{\text {n.s }}$ & $206^{\mathrm{n} . \mathrm{s}}$ & $16,54^{\mathrm{n} . \mathrm{s}}$ & $247^{\text {n.s }}$ & $4,5^{\text {n.s }}$ & $1,5^{\text {n.s }}$ & $4,2^{\mathrm{n} . \mathrm{s}}$ & $7,7^{\text {n.s }}$ & $1,7^{\mathrm{n} . \mathrm{s}}$ \\
\hline Resíduo & 30 & 3931 & 10217 & 1,3 & 4608 & 175 & 13,03 & 568 & 13,1 & 1,18 & 5,1 & 1,1 & 3,4 \\
\hline Média & & 621 & 1012 & 5,7 & 557 & 111 & 12,74 & 168 & 16,7 & 9,2 & 7,8 & 26,8 & 65,4 \\
\hline DP & & 99,3 & 179 & 1,3 & 96,9 & 25 & 4,98 & 22,60 & 3,5 & 1,3 & 2,8 & 1,2 & 2,2 \\
\hline $\mathrm{CV}_{\mathrm{e}}(\%)$ & & 10,1 & 9,99 & 20,1 & 12,2 & 11,8 & 28,32 & 14,11 & 21,7 & 11,8 & 28,9 & 3,8 & 2,8 \\
\hline
\end{tabular}

${ }^{*}$ Significativo no nível de $1 \%$ de significância; ${ }^{*}$ significativo no nível de $5 \%$ de significância; n.s = não significativo; DP = desvio padrão e $\mathrm{CV}_{\mathrm{e}}=$ coeficiente de variação experimental.

dos desbastes ou alguma característica específica do lenho, não foi suficiente para alterar essa propriedade da madeira. Outras pesquisas, no entanto, têm mostrado que tratamentos silviculturais podem acelerar o crescimento das árvores e ainda tendem a aumentar a densidade básica da madeira, o que pode ser verificado nos artigos de Wilkins \& Kitahara (1991), Malan \& Hoon (1992), Lima \& Garcia (2005) e Trevisan et al. (2007), em E. grandis. Contudo, Downes \& Raymond (1997), Lima et al. (2009) e Lima \& Garcia (2010) relataram que tal relação não é perfeita, ou seja, os tratamentos silviculturais que aumentam a produção de madeira nem sempre aumentam a densidade da madeira.

O valor médio obtido para a densidade básica $\left(621 \mathrm{~kg} \cdot \mathrm{m}^{-3}\right)$ está abaixo do que foi observado por Nogueira et al. (2008) para E. umbra de 15 anos (762 kg.m ${ }^{-3}$ ), plantado em Angatuba-SP, e para E. umbra de 25 anos (810 kg.m $\left.{ }^{-3}\right)$, plantado em Itapetininga-SP. Essas variações podem estar relacionadas às características edafoclimáticas do local do povoamento ou ser decorrentes de diferentes espaçamentos ou das características de solo ou genéticas, uma vez que há citações na literatura para espécies de outros gêneros, relacionando ambientes mais secos a maiores densidades (Barajas-Morales, 1985).

O comprimento de fibras diferiu significativamente entre as classes de diâmetro, sendo que a classe superior apresentou os maiores valores e a classe inferior, os menores. Embora as árvores tenham a mesma idade, essa diferença deve indicar que, naquelas de maiores diâmetros, o câmbio está mais maduro e com capacidade de produzir fibras mais longas do que nas árvores de menores diâmetros. Em trabalhos semelhantes com outras espécies do gênero, Rocha et al. (2004), para E. grandis, também observaram fibras mais longas na classe dominante com relação à classe dominada. Resultado diferente foi observado por Florsheim et al. (2009), em E. dunnii, em que a classe intermediária apresentou os maiores valores de comprimento de fibras e a classe dominada, os menores. Nesses dois estudos, as árvores apresentavam sete anos, sendo, portanto, mais jovens do que as estudadas no presente trabalho e, possivelmente, ainda não possuíam lenho adulto.

Da mesma forma que a densidade, os teores de lignina, holocelulose e extrativos também não diferiram entre as classes diamétricas. Como não foram encontrados parâmetros na literatura para E. umbra, cita-se o trabalho de Silva et al. (2007) para E. grandis, no qual o teor de extrativos foi superior, enquanto que os teores de lignina e holocelulose foram inferiores aos observados no presente estudo.

As características dos raios e vasos não mostraram diferenças significativas entre as classes diamétricas (Tabelas 1 e 2). Uma tendência diferente foi observada por Wilkes \& Abbott (1983), quando compararam árvores com crescimento rápido e lento para algumas espécies de Eucalyptus, sendo que as 
Tabela 2. Médias para densidade básica (DB), comprimento das fibras (CF), espessura da parede das fibras (EPF), comprimentos dos elementos de vaso $(\mathrm{CV})$, diâmetro dos vasos (DV), frequência dos vasos (FV), altura dos raios (AR), largura dos raios (LR), frequência dos raios (FR), teor de extrativo (TE), teor de lignina (TL) e teor de holocelulose (TH) de Eucalyptus umbra.

Table 2. Average of basic density (DB), fiber length (CF), fiber wall thickness (EPF), vessel element length (CV), vessel diameter (DV), vessel frequency (FV), ray height (AR), ray width (LR), ray frequency (FR), extractive content (TE), lignin content (TL) and holocellulose content (TH) of Eucalyptus umbra.

\begin{tabular}{|c|c|c|c|c|c|c|c|c|c|c|c|c|}
\hline Tratamento & $\begin{array}{c}\text { DB } \\
\left(\mathrm{kg} \cdot \mathrm{m}^{-3}\right)\end{array}$ & $\begin{array}{c}\mathrm{CF} \\
(\mu \mathrm{m})\end{array}$ & $\begin{array}{l}\text { EPF } \\
(\mu \mathrm{m})\end{array}$ & $\begin{array}{c}\text { CV } \\
(\mu \mathrm{m})\end{array}$ & $\begin{array}{c}\text { DV } \\
(\mu \mathrm{m})\end{array}$ & $\begin{array}{c}\text { FV } \\
\left(n^{\circ} \cdot \mathrm{mm}^{-2}\right)\end{array}$ & $\begin{array}{c}\text { AR } \\
(\mu \mathrm{m})\end{array}$ & $\underset{(\mu \mathrm{m})}{\mathbf{L r}}$ & $\begin{array}{c}\text { FR } \\
\left(n^{\circ} \cdot \mathrm{mm}^{-1}\right)\end{array}$ & $\begin{array}{l}\text { TE } \\
(\%)\end{array}$ & $\begin{array}{l}\text { TL } \\
(\%)\end{array}$ & $\begin{array}{l}\text { TH } \\
(\%)\end{array}$ \\
\hline Inferior & $631^{\mathrm{a}}$ & $960^{\mathrm{b}}$ & $5,4^{\mathrm{a}}$ & $540^{\mathrm{a}}$ & $113^{\mathrm{a}}$ & $13^{\mathrm{a}}$ & $162^{\mathrm{a}}$ & $15^{\mathrm{a}}$ & $9,1^{\mathrm{a}}$ & $9,0^{\mathrm{a}}$ & $26,2^{a}$ & $64,8^{\mathrm{a}}$ \\
\hline Mediana & $612^{\mathrm{a}}$ & $1009^{\mathrm{ab}}$ & $5,9^{\mathrm{a}}$ & $545^{\mathrm{a}}$ & $105^{\mathrm{a}}$ & $12^{\mathrm{a}}$ & $164^{\mathrm{a}}$ & $18^{\mathrm{a}}$ & $9,0^{\mathrm{a}}$ & $7,3^{\mathrm{a}}$ & $26,9^{a}$ & $65,8^{\mathrm{a}}$ \\
\hline Superior & $618^{\mathrm{a}}$ & $1065^{\mathrm{a}}$ & $5,7^{\mathrm{a}}$ & $585^{\mathrm{a}}$ & $116^{\mathrm{a}}$ & $12^{\mathrm{a}}$ & $179^{a}$ & $16^{\mathrm{a}}$ & $9,3^{\mathrm{a}}$ & $7,1^{\mathrm{a}}$ & $27,1^{\mathrm{a}}$ & $65,8^{\mathrm{a}}$ \\
\hline Posição radial (0\%) & $522^{\mathrm{b}}$ & $763^{c}$ & $4,5^{c}$ & $428^{b}$ & $75^{\mathrm{d}}$ & $19^{\mathrm{a}}$ & $162^{\mathrm{a}}$ & $18^{\mathrm{a}}$ & $10,7^{\mathrm{a}}$ & $6,9^{b c}$ & $27,3^{\mathrm{a}}$ & $65,8^{\mathrm{ab}}$ \\
\hline Posição radial (25\%) & $565^{\mathrm{b}}$ & $952^{\mathrm{b}}$ & $5,0^{\mathrm{bc}}$ & $545^{\mathrm{a}}$ & $105^{c}$ & $13^{\mathrm{b}}$ & $164^{\mathrm{a}}$ & $15^{\mathrm{a}}$ & $9,3^{\mathrm{ab}}$ & $8,0^{\mathrm{abc}}$ & $27,0^{\mathrm{a}}$ & $65,0^{\mathrm{b}}$ \\
\hline Posição radial (50\%) & $684^{\mathrm{a}}$ & $1054^{\mathrm{ab}}$ & $6,2^{\mathrm{ab}}$ & $579^{\mathrm{a}}$ & $114^{\mathrm{bc}}$ & $10^{\mathrm{b}}$ & $170^{\mathrm{a}}$ & $16^{\mathrm{a}}$ & $8,6^{\mathrm{b}}$ & $8,5^{\mathrm{ab}}$ & $26,1^{a}$ & $65,4^{\mathrm{ab}}$ \\
\hline Posição radial (75\%) & $741^{\mathrm{a}}$ & $1182^{\mathrm{a}}$ & $6,6^{\mathrm{a}}$ & $619^{\mathrm{a}}$ & $129^{\mathrm{ab}}$ & $9^{\mathrm{b}}$ & $176^{\mathrm{a}}$ & $16^{\mathrm{a}}$ & $8,4^{\mathrm{b}}$ & $10,3^{\mathrm{a}}$ & $26,2^{a}$ & $63,5^{\mathrm{b}}$ \\
\hline Posição radial (100\%) & $589^{b}$ & $1105^{\mathrm{a}}$ & $6,1^{\mathrm{abc}}$ & $613^{a}$ & $134^{\mathrm{a}}$ & $10^{\mathrm{b}}$ & $170^{\mathrm{a}}$ & $17^{\mathrm{a}}$ & $8,8^{\mathrm{b}}$ & $5,1^{\mathrm{c}}$ & $27,0^{\mathrm{a}}$ & $67,9^{a}$ \\
\hline
\end{tabular}

Obs.: Médias seguidas de letras diferentes em uma mesma coluna diferem entre si (no nível de 5\% de significância).

árvores que tiveram crescimento rápido apresentaram menor frequência de vasos. Rocha et al. (2004) verificaram que o lenho de árvores de E. grandis, aos sete anos da classe dominada (menor crescimento), apresentou maior frequência de vasos e as árvores da classe dominantes (maior crescimento), menor frequência de vasos. Florsheim et al. (2009) também verificaram, para E. dunnii de sete anos de idade, que a classe dominante apresentou menor frequência de vaso.

De maneira geral, a classe diamétrica influenciou apenas no comprimento das fibras, sem variação para as demais células, a densidade básica e os teores de extrativos, lignina e holocelulose. Tal fato pode ter ocorrido devido à origem das sementes e às suas características genéticas, justificando a homogeneidade da grande maioria das características. A pequena influência da taxa de crescimento nas dimensões celulares foi observada por Melo (1968), Malan (1991) e Malan \& Hoon (1992) em E. grandis.

$\mathrm{Na}$ variação radial, com exceção da altura e da largura dos raios e do teor de lignina, todas as demais variáveis mostraram diferenças significativas (Tabela 2).

No presente estudo, as maiores densidades foram observadas nas posições 50 e 75\%, e ocorreu uma redução brusca da densidade da posição $75 \%$ para a $100 \%$, a qual deve estar relacionada ao aumento do diâmetro dos vasos (Figura 3a), à diminuição na espessura da parede da fibra (Figura 2c) e ao teor de extrativos (Figura 5a). Em trabalhos com outras espécies de eucaliptos, Lima \& Garcia (2010), Polli et al. (2006) e Lima et al. (2009) observaram um incremento na densidade em direção da casca, sendo esse padrão o mais comum de ser observado nas espécies desse gênero; o aumento na densidade também estava associado ao aumento na espessura da parede das fibras.

Nas características anatômicas, o comprimento das fibras aumentou na direção da casca, comportamento similar ao da espessura da parede (Figura 2). O comprimento e o diâmetro dos vasos aumentaram no sentido medula-casca e a frequência diminuiu (Figuras 3a-3c). A altura e a largura dos raios não variaram e a frequência diminuiu na mesma direção (Figuras 4a-4c). Sugere-se que as variações das dimensões celulares podem ser devidas ao aumento gradativo da proporção de madeira adulta em relação à madeira juvenil no sentido medula-casca e, como mencionado, tais variações interferem diretamente na densidade da madeira $\mathrm{e}$, consequentemente, nas demais propriedades $\mathrm{e}$ aplicações.

Tomazello Filho et al. (1985), em um estudo com E. gummifera, E. microcorys e E. pilularis, concluiu que houve um aumento no comprimento das fibras no sentido medula-casca, atingindo valores acima de $1,0 \mathrm{~mm}$, a partir da posição $50 \%$ do raio. De um modo geral, a largura, o diâmetro do lume, e a 

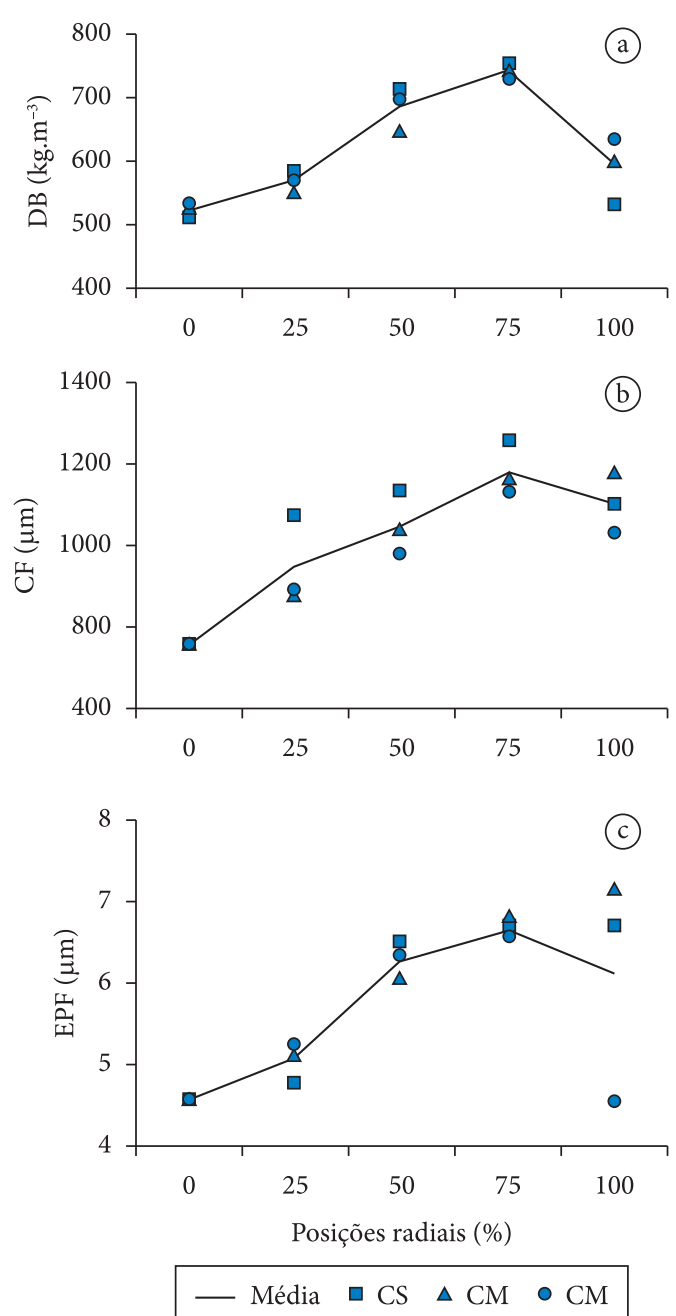

Figura 2. Variação radial da densidade básica e das dimensões de fibras. a) Densidade básica (DB); b) Comprimento da fibra (CF); c) Espessura da parede das fibras $(\mathrm{EPF}) . \mathrm{CS}=$ Classe superior, $\mathrm{CM}=$ Classe mediana, $\mathrm{CI}=$ Classe inferior.

Figure 2. Radial variation of basic density and fiber dimensions. a) Basic density (DB); b) Fiber length (CF); c) Fiber wall thickness (EPF). CS = Upper class, $\mathrm{CM}=$ Middle class, $\mathrm{CI}=$ Lower class.

espessura da parede das fibras, para as três espécies, tenderam ao aumento no sentido radial. Padrões semelhantes aos observados são mencionados para outras espécies do gênero Eucalyptus.

Rocha et al. (2004) verificaram para E. grandis que todas as dimensões das fibras apresentaram tendência crescente no sentido medula-casca; os vasos apresentaram tendência crescente para o comprimento e o diâmetro tangencial, e decrescente
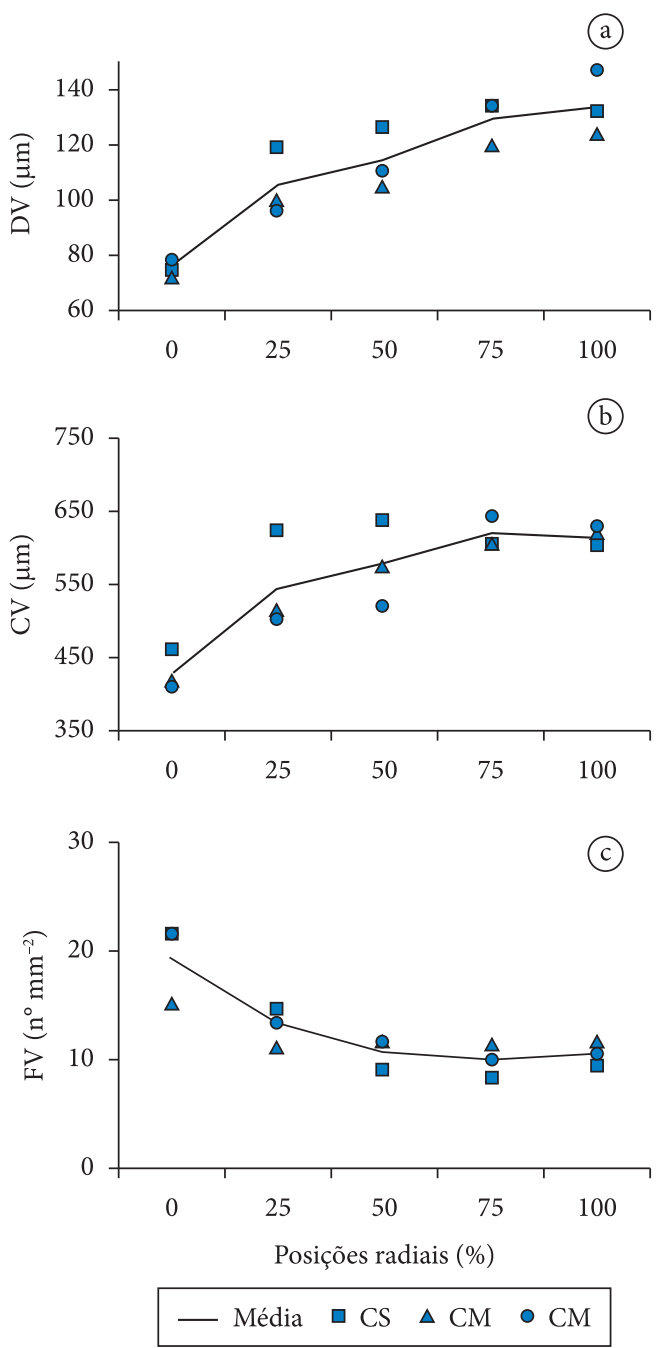

Figura 3. Variação radial das dimensões dos vasos. a) Diâmetro dos vasos (DV); b) Comprimentos dos elementos de vaso (CV); c) Frequência dos vasos (FV). $\mathrm{CS}=$ Classe superior, $\mathrm{CM}=$ Classe mediana, $\mathrm{CI}=$ Classe inferior.

Figure 3. Radial variation of vessel dimensions. a) Vessel diameter (DV); b) Vessel length (CV); c) Vessel frequency $(\mathrm{FV}), \mathrm{CS}=$ Upper class, $\mathrm{MC}=$ Middle class, $\mathrm{CI}=$ Lower class.

para a frequência, enquanto que, para a frequência de raios, a tendência foi decrescente. Quilhó et al. (2006) concluíram que as dimensões das fibras em clones de E. grandis $\times E$. urophylla não apresentaram tendência definida de variação no sentido medulacasca. Silva et al. (2007) observaram um aumento no comprimento das fibras no sentido medula-casca para E. grandis e a mesma tendência foi verificada para E. dunnii por Florsheim et al. (2009). Alzate (2009), em estudo semelhante, com as espécies 

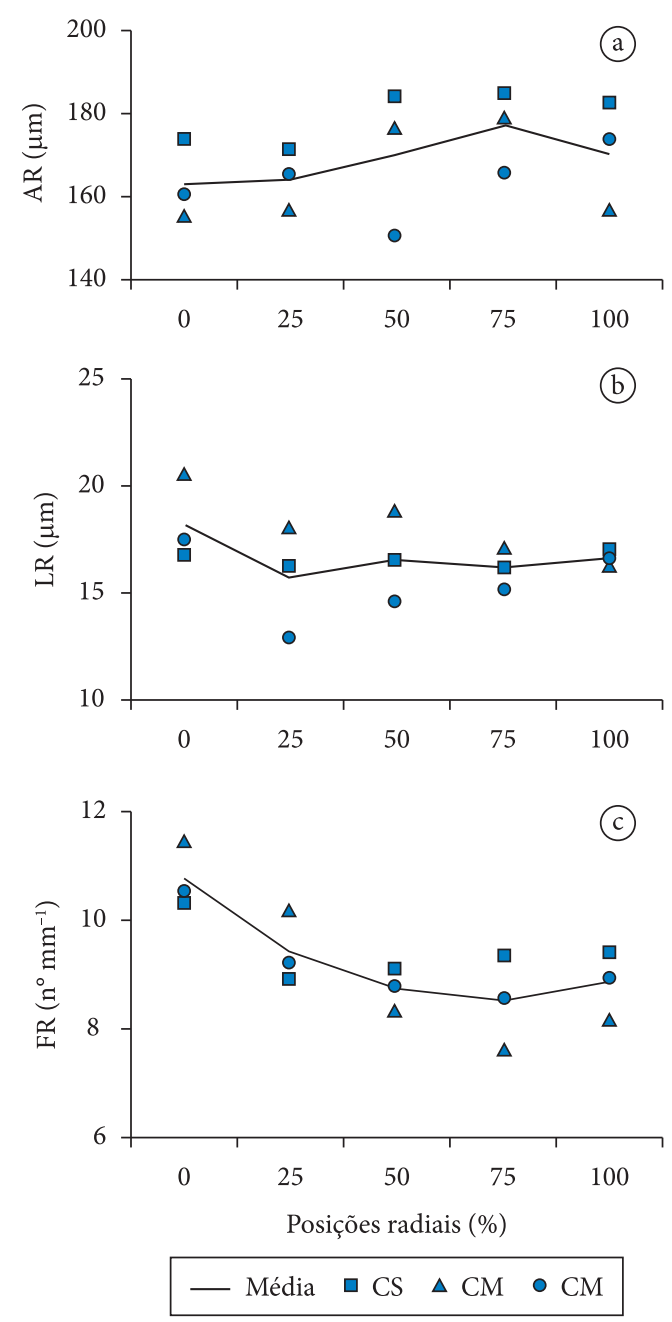

Figura 4. Variação radial das dimensões dos raios. a) Altura dos raios (AR); b) Largura dos raios (LR); c) Frequência dos raios (FR). CS = Classe superior, $\mathrm{CM}=$ Classe mediana, $\mathrm{CI}=$ Classe inferior.

Figure 4. Radial variation of ray dimensions. a) Ray height (AR); b) Ray width (LR); c) Ray frequency (FR). $\mathrm{CS}=$ Upper class, $\mathrm{MC}=$ Middle class, $\mathrm{CI}=$ Lower class.

E. grandis, E. saligna e E. grandis $\times$ E. urophylla, reportou aumento no comprimento e no diâmetro das fibras da medula até $75 \%$ do raio, e posterior tendência de estabilização; também verificou que houve aumento da espessura da parede e redução do lume das fibras até $50-75 \%$ do raio das amostras. Sette Júnior et al. (2009) verificaram para E. grandis que os vasos e as fibras mostraram os mesmos modelos radiais descritos para algumas espécies
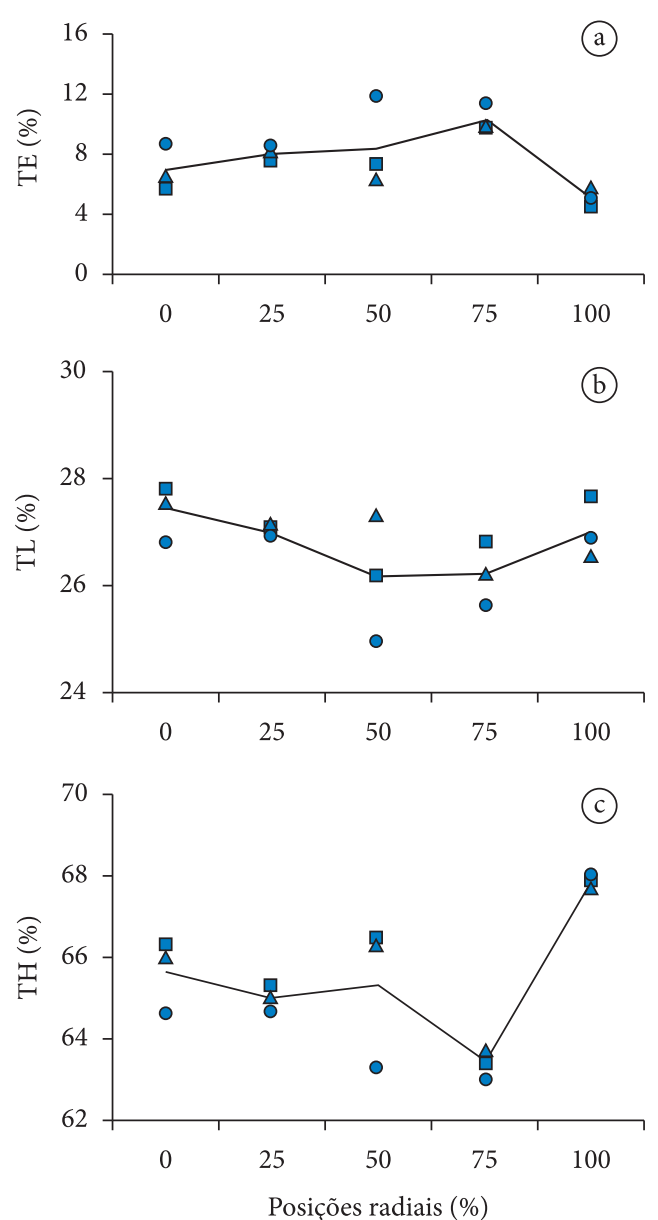

— Média $\square \mathrm{CS} \triangle \mathrm{CM}$ ○ $\mathrm{CM}$

Figura 5. Variação radial do teor dos constituintes químicos. a) Extrativo (TE); b) Lignina (TL); c) Holocelulose $(\mathrm{TH})$. $\mathrm{CS}=$ Classe superior, $\mathrm{CM}=$ Classe mediana, $\mathrm{CI}=$ Classe inferior.

Figure 5. Radial variation of the content of chemical compounds: a) Extractive (TE); b) Lignin (TL);c) Holocellulose. (TH) CS = Upper class, $\mathrm{MC}=$ Middle class, $\mathrm{CI}=$ Lower class .

de eucalipto. Ishiguri et al. (2009) verificaram essa mesma tendência para Paraserianthes falcataria.

Quanto aos componentes químicos, verificouse que o teor de lignina não mostrou variação radial, enquanto que a holocelulose e os extrativos apresentaram oscilações, sem nenhuma tendência de aumento ou diminuição no sentido medulacasca. Esse comportamento foi muito semelhante ao observado por Longui et al. (2010) para uma população de Piptadenia gonoacantha, com exceção 
do teor de extrativos que apresentou aumento significativo, no sentido medula-casca, enquanto que os teores de lignina e holocelulose não mostraram variação significativa.

Para entender melhor essas variações, foi realizado um estudo de relações entre as dimensões celulares com as posições radiais. Na Figura 6, são apresentados os modelos de regressões que melhor se ajustaram a essas variações significativas. De
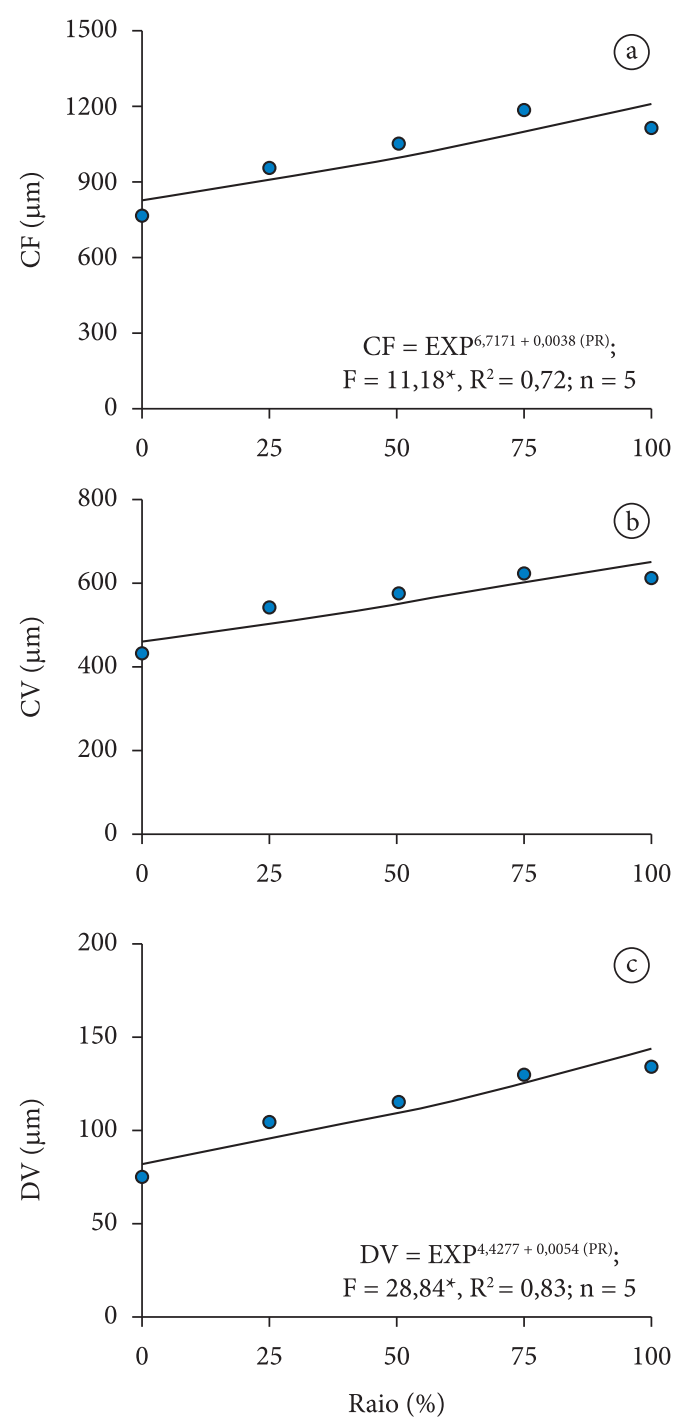

Figura 6. Relações entre posição radial (PR) e características anatômicas. a) Comprimento das fibras (CF); b) Comprimentos dos elementos de vaso (CV); c) Diâmetro dos vasos (DV) em Eucalyptus umbra. ${ }^{*} \mathrm{P}=0,01 \mathrm{e}^{*} \mathrm{P}=0,05$.

Figure 6. Relationships between radial positions (PR) and anatomical features. a) Fiber length (CF); b) Vessel element length $(\mathrm{CV})$; c) Vessel diameter (DV) in Eucalyptus umbra. ${ }^{\star *} \mathrm{P}=0.01$ and ${ }^{\star} \mathrm{P}=0.05$. acordo com os modelos de regressão linear obtidos, verifica-se um aumento significativo, no sentido medula-casca, somente para o comprimento das fibras e o comprimento e o diâmetro dos vasos, o que possivelmente reflete a maturação do câmbio vascular.

\section{CONCLUSÕES}

A classe de diâmetro apresentou pouca influência nas propriedades estudadas. Somente o comprimento de fibras diferiu significativamente entre as classes de diâmetro, sendo que a classe superior apresentou os maiores valores e a classe inferior, os menores.

A largura e a frequência dos raios e o teor de lignina total não foram influenciados significativamente pela posição radial.

O comprimento das fibras, assim como o comprimento e o diâmetro dos vasos, aumentaram significativamente no sentido medula-casca. Sugerese que as variações das dimensões celulares podem ser causadas pelo aumento gradativo da proporção de madeira adulta em relação à madeira juvenil no sentido medula-casca.

\section{AGRADECIMENTOS}

Os autores agradecem pelo auxílio laboratorial de Sonia Godói Campião, da Seção de Madeira e Produtos Florestais do Instituto Florestal, e à Maria Regina Buch do Laboratório de Química, Celulose e Energia da ESALQ/USP.

\section{STATUS DA SUBMISSÃO}

Recebido: 18/07/2011

Aceito: 23/09/2011

Resumo publicado online: 25/08/2011

Artigo completo publicado: 30/09/2011

\section{AUTOR(ES) PARA CORRESPONDÊNCIA}

\section{Israel Luiz de Lima}

Divisão de Dasonomia,

Instituto Florestal do Estado de São Paulo,

Rua do Horto, 931, CEP 02377-000,

São Paulo, SP, Brasil

e-mail: israelluizde.lima@yahoo.com.br 


\section{REFERÊNCIAS}

Alzate BA. Estrutura anatômica da madeira de clones de Eucalyptus. Revista Investigaciones Aplicadas 2009; 5:1-14.

Barajas-Morales J. Wood structural differences between trees of two tropical forests in Mexico. IAWA Bulletin $1985 ; 6: 355-364$.

Berlyn, GP, Miksche, JP. Botanical microtechnique and cytochemistry. The Iowa University Press, Iowa. 1976. $326 \mathrm{p}$.

Cruz CR, Lima JT, Muniz GIB. Variações dentro das árvores e entre clones das propriedades físicas e mecânicas de híbridos de Eucalyptus. Scientia Forestalis 2003; 64:33-47.

Downes GM, Raymond CA. Variation in wood density in plantation eucalypts. In Downes GM, Hudson I, Raymond CA, Dean GH, Michell AJ, Schimleck LR. et al., editors. Sampling Plantation Eucalypts for Wood and Fibre Properties. Melbourne: CSIRO Publishing; 1997. appendix 1, p. 88-99.

Florsheim SMB, Couto HTZ, Lima IL, Longui EL. Variação nas dimensões dos elementos anatômicos da madeira DE Eucalyptus dunnii aos sete anos de idade. Revista do Instituto Florestal 2009; 21(1):79 - 91.

Hall N, Brooker I. Broad-leaved white mahogany. Melbourne: CSIRO, Forest Tree Series; 1977. n. 92, p. 3.

International Association of Wood Anatomists Committee - IAWA list of microscopic features for hardwood identification. IAWA Bulletin 1989; 10(3):219-332.

Ishiguri F, Hiraiwa T, Iizuka K, Yokota S, Priadi D, Sumiasri N. et al. Radial variation of anatomical characteristics in Paraserianthes falcataria planted in Indonesia. IAWA Journal 2009; 30(3):343-352.

Latorraca JVF, Albuquerque CEC. Efeito do rápido crescimento sobre as propriedades da madeira. Floresta e Ambiente 2000; 7(1):279-91.

Lima IL, Florsheim SMB, Longui EL. Influência do espaçamento em algumas propriedades físicas da madeira de Tectona grandis Linn. Cerne 2009; 15(2):244-250.

Lima IL, Garcia JN. Variação da densidade aparente e resistência à compressão paralela às fibras em função da intensidade de desbaste, adubação e posição radial em Eucalyptus grandis Hill ex-maiden. Revista Árvore 2010; 34(3):551-559.

Lima IL, Garcia JN. Influência do desbaste em propriedades físicas e mecânicas da madeira de Eucalyptus grandis Hill ex-Maiden. Revista do Instituto Florestal 2005; 17(2):151-160.
Longui EL, Lima IL, Silva FG, Bufolo A, Suckow IMS, Florsheim SMB. Variação radial das características anatômicas, densidade aparente, teores de extrativos, lignina e holocelulose na madeira de Piptadenia gonoacantha (Mart.) Macbr. Scientia Forestalis 2010; 38:341-353.

Longui EL. Pau-brasil e outras espécies nativas empregadas na confeç̧ão de arcos para instrumentos de corda: um estudo comparativo [dissertação]. São Paulo: Instituto de Botânica da Secretaria de Estado do Meio Ambiente; 2005.

Lorenzi H, De Souza HM,Torres MAV, Bacher LB. Árvores exóticas no Brasil: madeireiras, ornamentais e aromáticas. Nova Odessa: Instituto Plantarum; 2003. $368 \mathrm{p}$.

Malan FS., Variation, association and inheritance of juvenile wood properties of Eucalyptus grandis Hill ex Maiden with special reference to the effect of rate of growth. South African Forestry Journal 1991; 157:16-23. http://dx.doi.org/10.1080/00382167.1991.9629094

Malan FS, Hoon M. Effect of initial spacing and thinning on some wood properties of Eucalyptus grandis. South African Forestry Journal 1992; 163:13-20. http://dx.doi. org/10.1080/00382167.1992.9629362

Mello HA. Efeitos da adubação mineral sobre as qualidades da madeira. Piracicaba: IPEF/ESALQ, 1968. 15 p. Relatório Técnico.

Nogueira AAP, Aoki H, Calil Junior C. Determinação das características físico-mecânica do Eucalyptus umbra. In Anais do Encontro Brasileiro em Madeiras e Estruturas de Madeiras; 2008; Londrina. Londrina: EBRAMEM; 2008.

Panshin AJ, De Zeeuw C. Textbook of Wood Technology: structure, identification, properties and uses of the commercial woods of the United States and Canada. 3rd ed. Nova York: McGraw-Hill; 1964. 643 p.

Polli HQ., Reis GG, Reis MGF, Vital BR, Pezzopane JEM, Fontan ICI. Qualidade da madeira em clone de Eucalyptus grandis W. Hill ex Maiden submetido a desrama artificial. Revista Árvore 2006; 30(4):557-566.

Quilhó T, Miranda I, Pereira H. Within-tree variation in wood fibre biometry and basic density of the urograndis eucalypt hybrid (Eucalyptus grandis $\times$ E. urophylla). Iawa Journal 2006; 27:243-254.

Rocha FT, Florsheim SMB, Couto HTZ. Variação das dimensões dos elementos anatômicos da madeira de árvores de Eucalyptus grandis hill ex maiden aos sete anos. Revista do Instituto Florestal 2004; 16(1):43-55.

SAS Institute. SAS Procedures Guide. Version 8 (TSMO). Cary: SAS Institute Inc.; 1999.

Sass JE. Botanical Microtechniche. Ames: The Iowa State College Press; 1951.326 p. 
Sette Júnior CR, Tomazello Filho M, Dias CTS, Chagas MP, Laclau JP. Efeito da aplicação de potássio e sódio nas características do lenho de árvores de Eucalyptus grandis W. Hill, aos 24 meses de idade. Floresta 2009; 39(3):535-546.

Silva JC, Matos JLM, Oliveira JTS, Evangelista WV. Influência da idade e da posição ao longo do tronco na composição química da madeira de Eucalyptus grandis Hill ex. Maiden. Revista Árvore 2007; 29(3):455-460.

The Leading Association for the Worldwide Pulp, Paper, Packaging and Converting Industries - TAPPI. T222 om-98 Acid-insoluble lignin in wood and pulp. Norcross: TAPPI Test Methods; 1998.

Tomazello Filho M, Barrichelo LEC, Costa JC. Análise da madeira de compressão em Pinus oocarpa e Pinus strobus var. chiapensis. composição química. Revista IPEF 1985; 31:69-73.

Trevisan R, Haselein CR, Santini EJ, Schneider PR, Menezes LF. Efeito da intensidade de desbaste nas características dendrométricas e tecnológicas da madeira de Eucalyptus grandis. Ciência Florestal 2007; 17(4):377-387.

Varguese M, Nicodemus A, Ramteke PK, Anbazhagi G, Bennet SSR, Subramanian K. Variation in Growth and Wood Traits Among Nine Populations of Teak in Peninsular Índia. Silvae Genetica, 2000; 49(2-5):201205.

Vital BR. Métodos de determinação da densidade da madeira. Boletim Técnico Sociedade de Investigações Florestais 1984; (1):1-21.

Wilkes J, Abbott D. Influence of the rate of tree growth on the anatomy of eucalypt species. Appita 1983; 37(3):231-231.

Wilkins AP, Kitahara R. Silvicultural treatments and associated growth rates, growth strains and wood properties in 12.5-year-old Eucalyptus grandis. Australian Forestry 1991; 54(1-2):99-104.

Zobel BJ, Buijtenen JV. Wood variation, its causes and control. Berlin: Springer; 1989. 363 p. 